\title{
Conversion of Diffusely Abnormal White Matter to Focal Lesions is Linked to Progression in Secondary Progressive Multiple Sclerosis
}

Mahsa Dadar, $\mathrm{PhD}^{1,2}$, Sridar Narayanan, $\mathrm{PhD}^{1}$, Douglas L. Arnod, $\mathrm{MD}^{1}$, D Louis Collins, $\mathrm{PhD}^{1,2}$, Josefina Maranzano, $\mathrm{MD}, \mathrm{PhD}^{1,3}$

(1) McConnell Brain Imaging Centre, Montreal Neurological Institute, McGill University, Montreal, Quebec, Canada

(2) Department of Biomedical Engineering, McGill University, Montreal, Quebec, Canada

(3) Department of Anatomy, University of Quebec in Trois-Rivieres, Trois-Rivieres, Quebec, Canada

Corresponding author: Josefina Maranzano, MD, $\mathrm{PhD}$.

josefina.maranzano@uqtr.ca

josefina.maranzano@mcgill.ca

- University of Quebec in Trois-Rivieres, Department of Anatomy. Pavillon LeonProvancher. Local 3501. 3351, boulevard des Forges, Trois-Rivieres, Quebec, Canada. G8Z 4M3.

- McGill University, Department of Neurology and Neurosurgery, Montreal Neurological Institute. 3801 Rue University, Room WB327, Montreal, Quebec, Canada, H3A 2B4.

\section{STUDY FUNDING}

This Investigation was supported (in part) by (an) award(s) from the International Progressive MS Alliance, award reference number PA-1603-08175 


\begin{abstract}
Objectives: 1) To automatically segment focal white matter lesions (FWML) and Diffusely abnormal white matter (DAWM), i.e. regions of diffuse abnormality observed on conventional (T2-weighted) MRI and characterize their longitudinal volumetric and normalized T1-weighted (T1w) intensity evolution, 2) To assess associations of FWML and DAWM with Expanded Disability Status Scale (EDSS) and confirmed disability progression (CDP).
\end{abstract}

Methods: Data includes 3951 timepoints of 589 SPMS participants followed for three years. FWML and DAWM were automatically segmented using a 2-weighted-intensity thresholding technique. Screening DAWM volumes that transformed into FWML at the last visit (DAWM-toFWML) and normalized T1w intensities (as a marker of severity of damage) in those voxels were calculated.

Results: FWML volume significantly increased and DAWM volume significantly decreased as disease duration increased $(\mathrm{p}<0.001)$. Global EDSS scores were positively associated with FWML volumes $(\mathrm{p}=0.002)$, but not with DAWM volumes. Median volume of DAWM-to-FWML was significantly higher in patients who progressed (2.75 vs $1.70 \mathrm{cc} ; \mathrm{p}<0.0001)$, and represented $14 \%$ of the total DAWM volume at screening, compared to $10 \%$ in patients who did not progress $(\mathrm{p}=0.001)$. Normalized T1w intensity values of DAWM-to-FWML were negatively associated with CDP status $(\mathrm{p}<0.00001)$.

Conclusion: DAWM transformed into FWML over time, and this transformation was significantly associated with clinical progression. DAWM voxels that transformed had greater normalized T1w intensity decrease over time, in keeping with relatively greater tissue damage evolution. Evaluation 
of DAWM in progressive MS provides a useful measure to evaluate therapies that aim to protect this at-risk tissue with the potential to slow progression.

Keywords: Secondary Progressive Multiple Sclerosis, Diffusely Abnormal White Matter, Focal White Matter Lesions, Progression

\section{INTRODUCTION}

Multiple Sclerosis (MS) is an inflammatory and neurodegenerative demyelinating disease characterized by lesions that affect the white matter (WM) and gray matter (GM) of the central nervous system $(\mathrm{CNS})^{1}$. The inflammatory component of the disease is more prominent in the early phases and is characterized by disruption of the blood-brain-barrier (BBB) and lymphocytic infiltration that leads to myelin and axonal damage in a focal area traditionally described as the MS plaque ${ }^{1}$. In most patients (approximately 80\%), the inflammatory episodes subside and these focal white matter lesions (FWML) repair either partially or completely ${ }^{1}$. This early phase of MS is called relapsing remitting MS (RRMS), clinically translating into successive episodes of symptoms that spontaneously regress with varying degrees. As the disease evolves, the accumulation of these episodes and their incomplete recovery lead to an accumulation of lesions and progressive loss of axons, which is considered the neurodegenerative hallmark of MS, more prominent in the later stages ${ }^{2,3}$. This later phase clinically translates into an accumulation of symptoms without, or un-related to, relapses and it is known as secondary progressive MS $(\mathrm{SPMS})^{4}$.

During the last three decades, RRMS has been studied in depth, and its inflammatory component, in the form of FWML, has been characterized in detail, using MRI and histopathology data ${ }^{3,5}$. On 
MRI, focal lesions are relatively straightforward to detect and quantify; they appear as discrete areas of high signal intensity on $\mathrm{T} 2$ weighted (T2w) and fluid attenuated inversion recovery (FLAIR) images, and low signal intensity on T1 weighted (T1w) MRI contrasts ${ }^{5}$. Currently, there are multiple therapies that target and successfully control the development of inflammatory FWML in $\mathrm{MS}^{6}$.

Unfortunately, the pathophysiological mechanisms of SPMS are more elusive, and different types of lesions have been proposed as candidates that associate more significantly with progression and disability: cortical lesions $(\mathrm{CLs})^{7}$, brain atrophy ${ }^{8}$, and diffusely abnormal WM (DAWM) ${ }^{7,9,10}$. On T2w MRI, the latter consists of mildly hyperintense areas with poorly defined edges of an intermediate value between that of normal-appearing WM (NAWM) and FWML. In histopathology, DAWM and FWML show differences in the degree of demyelination, axonal loss, and immune cell density, which are all more severe in FWML ${ }^{11}$.

Previous studies have shown a high correlation between normalized T1w intensity values and magnetization transfer ratio (MTR) values, which in turn have been shown to be sensitive to myelin content $^{12}$. Hence, T1w-normalized intensities can be used as a proxy of MTR to roughly reflect the degree of demyelination of a given area: the lower the intensity value, the greater the myelin loss.

Our study takes advantage of a previously validated automated method to segment FWML and DAWM $^{12}$ on scans of SPMS participants who were part of a three-year longitudinal study ${ }^{13}$. We describe the evolution of the volumes and the T1w-normalized intensity values of these two regions and explore their relationships with brain atrophy and progression of symptoms. 


\section{METHODS}

\subsection{Study Design}

Our study is a retrospective, longitudinal, observational analysis to determine the volume and T1wnormalized intensity values of FWML and DAWM in SPMS participants ${ }^{13}$.

\subsection{Population}

Data used in this study is part of the International Progressive Multiple Sclerosis Alliance (IPMSA) study. We included MRI scans of 589 SPMS participants, enrolled in an unsuccessful clinical trial (no improvement of progression during the length of the trial), scanned longitudinally for 3 years, at screening, as well as weeks $24,48,72,96,108$, and 156 . The total number of timepoints included in this study was $3951^{13}$. All subjects had 3D T1w and 2D T2w images available. Table 1 summarizes the acquisition parameters for the $\mathrm{T} 1 \mathrm{w}$ and $\mathrm{T} 2 \mathrm{w}$ scans.

Table 1. MRI acquisition parameters. $\mathrm{TR}=$ Repetition time. $\mathrm{TE}=$ Echo time. $\mathrm{TI}=\mathrm{Inversion}$ time. $\mathrm{T} 1 \mathrm{w}=\mathrm{T} 1$-weighted. $\mathrm{T} 2 \mathrm{w}=\mathrm{T} 2$-weighted. $\mathrm{TE}, \mathrm{TR}$, and Flip angle are expressed as ranges, to encompass all values used by different sites.

\begin{tabular}{|l|l|l|}
\hline & T1w & T2w \\
\hline Sequence & 3D Flash & 2D TSE \\
\hline Orientation & axial & axial \\
\hline TR (ms) & $20-30$ & $4800-6100$ \\
\hline TE (ms) & $3-11$ & $78-89$ \\
\hline Flip Angle & $27-30$ & $90-180$ \\
\hline Number of slices & 60 & 60 \\
\hline Voxel size $(\mathrm{mm})$ & $1 \times 1 \times 3$ & $1 \times 1 \times 3$ \\
\hline
\end{tabular}




\subsection{Image Processing}

All MR images were processed using the following steps:

1. Image intensity non-uniformity correction using N3 $3^{14}$.

2. Linear image intensity normalization into range [0-100].

3. Brain tissue extraction using brain extraction based on nonlocal segmentation technique $(\mathrm{BEaST})^{15}$

4. Tissue classification in the native space of the $\mathrm{T} 1 \mathrm{w}$ images using a tissue segmentation pipeline based on a random forests classifier $(\mathrm{BISON})^{16}$.

5. Rigid co-registration of the $\mathrm{T} 2 \mathrm{w}$ images to the $\mathrm{T} 1 \mathrm{w}$ images of the same timepoint. Using these transformations, the tissue masks and brain masks were also transformed to the native T2w space.

6. Linear registration (9 parameters) of the T1w images to MNI-ICBM152 template ${ }^{17}$.

7. Nonlinear registration of the T1w images to MNI-ICBM152 template ${ }^{18}$.

8. Generation of deformation-based morphometry (DBM) maps using MINC tools.

9. Using an atlas of the cortical and subcortical GM regions ${ }^{19,20}$, average Jacobian determinant values from the DBM deformation field were calculated as estimates of regional atrophy/change for each GM region.

10. Automatic segmentation of WM lesions using a Bayesian Classifier ${ }^{21}$.

All of the generated masks and registrations were visually assessed and those that did not pass this quality control (QC) were excluded from the analyses. All of the pipelines used in the processing of the images have been developed and validated for use in multi-center and multi-scanner datasets and have been previously used in many such applications ${ }^{17,16,22}$. 


\subsection{Manual Selection of Thresholds to Separate FWML and DAWM}

Sixty-seven scans (timepoints) were selected from the dataset. To ensure the generalizability of the results, the subjects were selected to have MRIs from different scanner models, of three manufacturers (Achieva from Philips, Sonata, Symphony, and Avanto from SIEMENS, and SIGNA and DISCOVERY from GE). An expert rater (JM), reviewed the co-registered sequences for each timepoint and selected 2 T2w intensity threshold values to separate the NAWM from DAWM and FWMLs. These values were used to estimate the regression weights for the automatic thresholding technique for the entire dataset (see step 2.4 below).

\subsection{Automatic Segmentation of FWML and DAWM}

The main contrast used for separation of FWML and DAWM from NAWM tissue was the T2w image. To achieve this, the co-registered WM masks obtained from the tissue classification step were eroded by one voxel (in the axial plane) to avoid any partial volume effects between tissue borders (ventricular fluid and cortical GM). An initial NAWM mask was generated by excluding the WM lesions, generated with the Bayesian classifier, from this eroded WM mask. The mode of the intensity histogram of the T2w image within this initial NAWM was calculated. Previous work has shown that the manually selected thresholds and this automatically determined mode value have a linear relationship ${ }^{12}$. Using a linear regression, the optimal weights for estimating DAWM and FWML threshold values based on these automatically generated modes were calculated. Based on this criterion, any voxels within the eroded WM mask with intensities higher than 1.45 times the mode of the intensity histogram were labeled as FWML. Similarly, any voxels within the eroded WM mask in the range [1.15-1.45] times the mode of the intensity histogram were labeled 
as DAWM. The remaining tissue was labeled as NAWM. All of the separations were visually assessed by JM and those that did not pass this quality control were excluded ( $n=84$ scans: $2 \%$ ).

\subsection{T1w-normalized Intensity Values in DAWM and FWML Areas}

T1w-normalized values were calculated for the two areas of interest (DAWM and FWML) using the mode of the histogram of NAWM as the normalization factor. Due to the high correlation between MTR values and normalized T1w intensities, these two T1w-normalized intensities are adequate to reflect the degree of demyelination of a given area: the lower the intensity value, the greater the myelin loss.

\subsection{Conversion of Lesion Types}

Using the longitudinally registered first and last scans of each participant, FWML and DAWM masks of the two visits were compared, and voxels that changed from one mask type to the other between the two visits (e.g. from NAWM or DAWM to FWML) were identified (i.e. as NAWMto-FWML, or DAWM-to-FWML). These labels were then propagated across all available timepoints and their T1w-normalized intensities were calculated.

\subsection{Clinical variables}

All participants were assessed for the following variables at the corresponding scan visit: 1) Global Expanded Disability Status Scale (EDSS) and its sub-scores (e.g. Cerebellar functional system), 2) Symbol Digit Modalities Test (SDMT), 3) 9-Hole Peg Test (9HPT), and 4) determination of confirmed disability progression (CDP) for 12 and 24 weeks. CDP was defined as an increase of the global EDSS score that remained stable for at least 12 or 24 weeks. The increase had to be of 
1 point if the baseline EDSS was between 0 and 5.5, or 0.5 if the baseline EDSS was equal to or greater than 6.

\subsection{Statistical Analysis}

Demographic and MRI variables are presented using descriptive statistics, as either mean and standard deviation (SD), or median and range, according to their distribution. Differences in volumes and T1w normalized intensities of DAWM and FWML were assessed using relatedsample Wilcoxon Signed Rank Test. Differences in volumes of DAWM-to-FWML, NAWM-toFWML, and NAWM-to-DAWM between patients with CDP for 12 and 24 weeks (progressors) and patients without CDP (non-progressors) were assessed using Mann-Whitney U test.

Longitudinal mixed effects models were used to test the following hypotheses:

i. FWML and DAWM volumes change as a function of Disease Duration and Age at Onset.

ii. GM atrophy is associated with FWML and DAWM volume changes.

iii. Worsening clinical symptoms and progression are associated with FWML and DAWM volume changes.

iv. Longitudinal changes of mean T1w intensity values in DAWM-to-FWML voxels (DAWM areas on the first scan that become FWML on the last scan) are associated with progression.

DAWM and FWML volumes were log-transformed to achieve normal distributions. All continuous variables were z-scored prior to the analyses. Longitudinal mixed effects models were used to study the association between DAWM and FWML lesion volumes, regional GM atrophy measures, and clinical variables. All models included age at disease onset, disease duration, sex, and treatment (treatment did not have a significant effect in any of the analyses). Subject was entered as a categorical random variable. The regional GM-DBM results were corrected for 
bioRxiv preprint doi: https://doi.org/10.1101/832345; this version posted November 7, 2019. The copyright holder for this preprint (which was not certified by peer review) is the author/funder, who has granted bioRxiv a license to display the preprint in perpetuity. It is made available under aCC-BY-NC-ND 4.0 International license.

multiple comparisons using false discovery rate (FDR $)^{23}$ correction with a significance threshold of 0.05. All analyses were performed in MATLAB version R2017b.

\section{RESULTS}

From a total of 4035 scans, 84 were excluded due to misregistration of the T2w to the T1w image (2\%). Figure 1 shows an example of the segmentation of DAWM and FWML for one subject. The first row shows axial slices of the $\mathrm{T} 2 \mathrm{w}$ image. The second row shows the automatic tissue classification and WM lesion masks overlaid on the T2w image. The third row shows the automatically generated DAWM and FWML masks overlaid on the T1w images.
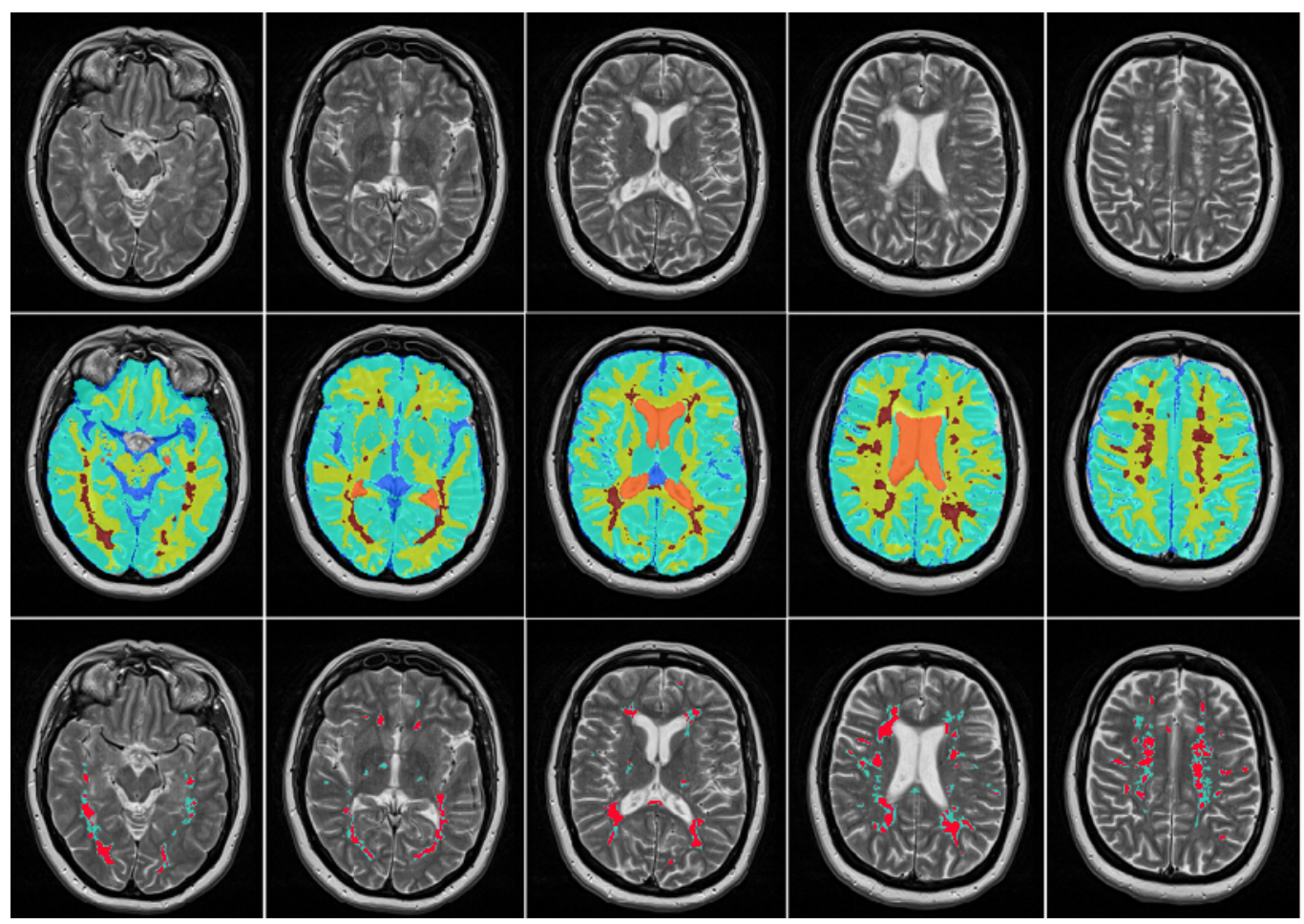

T2w Image
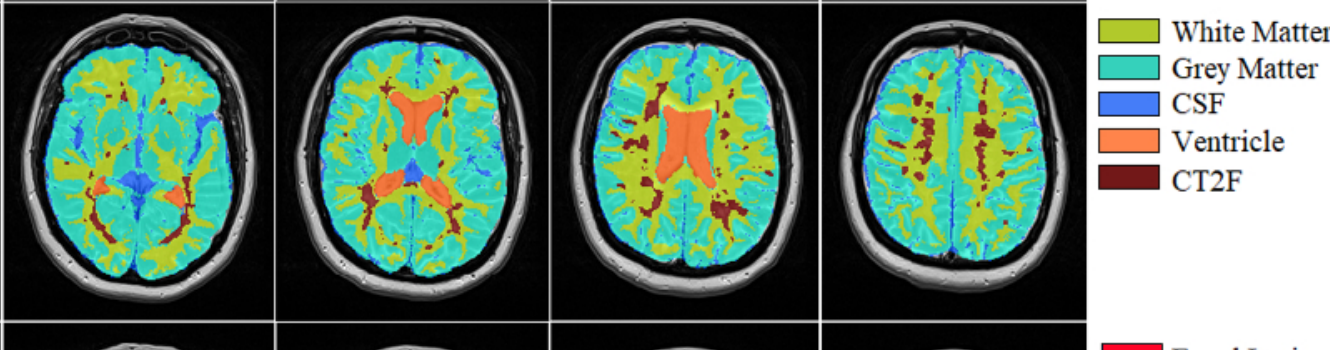

$\square \mathrm{CSF}$

$\square$ Ventricle

CT2F
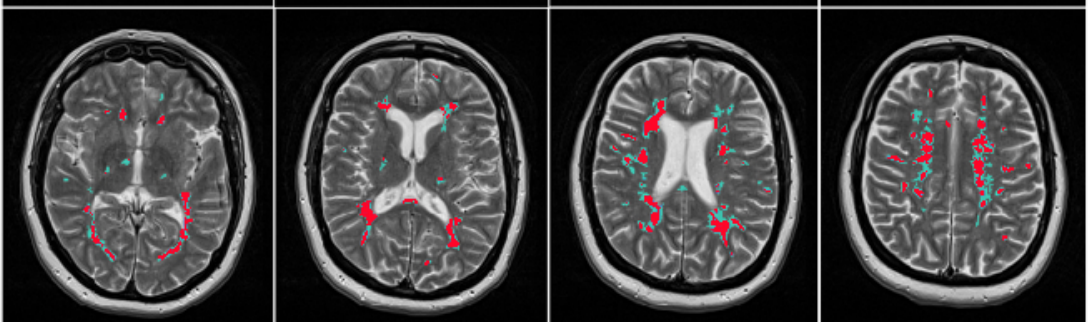

Focal Lesions DAWM

Figure 1. Example of the separation of DAWM and FWML. First row: T2w image. Second row: tissue classification and WM lesion masks on the T2w image. Third row: automatically generated 
DAWM and FWML masks on the T1w images. DAWM= Dirty Appearing White Matter. FWML= Focal White Matter Lesion. CSF: cerebrospinal fluid. CT2F: Bayesian lesion mask.

\subsection{Population}

The mean age of the participants at the time of the first scan was $49.24 \pm 7.36$ years, with a mean overall disease duration of $17.63 \pm 7.32$ years, and a duration of the secondary progressive phase of $6.38 \pm 3.53$ years. Table 2 summarizes the demographic characteristics of the participants.

Table 2. Demographic characteristics of the participants included in this study. Values represent number and percentage or mean \pm standard deviation. RRMS $=$ Relapsing Remitting Multiple Sclerosis. SPMS= Secondary Progressive Multiple Sclerosis. EDSS= Expanded Disability Status Scale.

\begin{tabular}{|l|l|}
\hline Sex & $245 \mathrm{M}(36 \%)$ \\
\hline Age at the time of scan & $49.24 \pm 7.36$ \\
\hline Age at the onset of RRMS (age at first symptom) & $31.61 \pm 8.05$ \\
\hline Age at the onset of SPMS & $42.86 \pm 7.34$ \\
\hline Disease duration & $17.63 \pm 7.32$ \\
\hline SPMS duration & $6.38 \pm 3.53$ \\
\hline EDSS & $5.69 \pm 1.10$ \\
\hline
\end{tabular}




\subsection{MRI Variables}

The median FWML volume in the dataset was 10.21cc (range: 0.03-13.61cc). The median DAWM volume was $21.59 \mathrm{cc}$ (range: $0.87-95.19 \mathrm{cc}$ ) and it was significantly higher than the FWML volume $(\mathrm{p}<0.0001)$. The median FWML T1w-normalized intensity was 0.83 (range $0.00-1.00$ ). The median DAWM T1w-normalized intensity was 0.96 (range: 0.01-1.01) and it was significantly higher than the FWML T1w-normalized intensity $(\mathrm{p}<0.0001)$.

\subsection{Longitudinal FWML and DAWM Volume Changes}

Mixed effects models were used to assess the relationship between FWML and DAWM volumes and Age of Onset and Disease Duration, controlling for Sex, and Treatment. Both FWML and DAWM volumes were significantly and negatively associated with Age at Onset: the older the person, the lower the volume of these two types of hyperintensity $(\mathrm{p}<0.0001$ for both FWML and DAWM) (Figure 2, note use of ordinate log scale). FWML volume significantly increased $(p=0.001)$ and DAWM volume significantly decreased $(p<0.0001)$ as Disease Duration increased

(Figure 2). Additionally, the ratio of FWML/DAWM volume per-participant significantly increased $(\mathrm{p}<0.0001)$ with Disease Duration (Figure 2). 

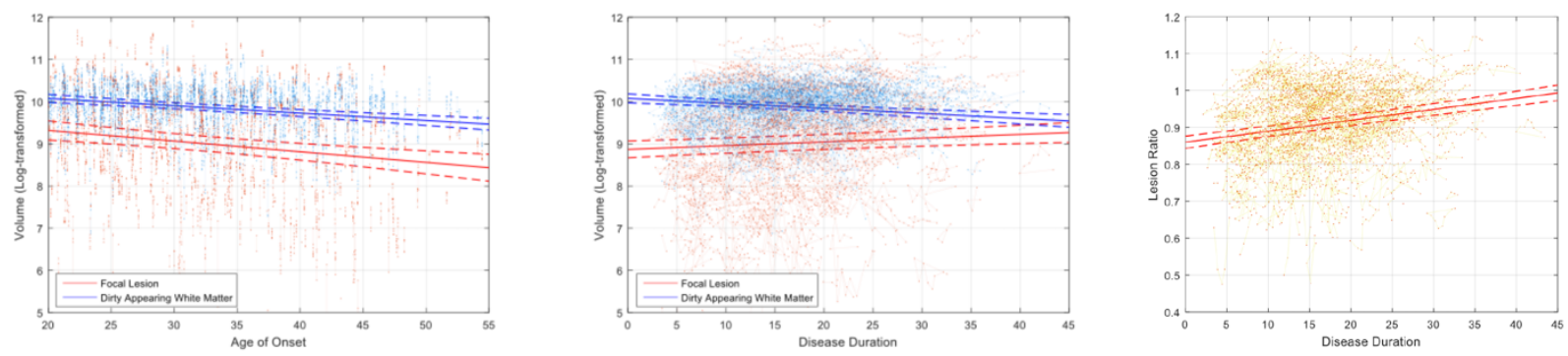

Figure 2: Left panel shows the volumes of DAWM (in blue) and FWML (in red) in relation to the age at disease onset (age at first symptom). Mid-panel shows the volumes of DAWM (in blue) and FWML (in red) in relation to the overall disease duration (from age at first symptom until the age at the time of the scan). Right panel: shows the Volume Ratio of FWML/DAWM in relation to disease duration

\subsection{GM Atrophy and FWML and DAWM Volumes}

Mixed effect models were used to assess the relationship between regional GM atrophy and FWML and DAWM volumes, controlling for Age, Sex, and Treatment. Figure 3 shows t-statistics maps of the GM regions that remained significant after FDR correction. Atrophy in both thalami, putamen, pallidum, brainstem, and cortical cerebellar and cerebral cortical GM were significantly associated with FWML volume increase (Figure 3). In parallel, cortical areas of right occipital lobe, right temporal lobe, left insular lobe, cerebellum, and brainstem were significantly associated with DAWM volume decrease (Figure 3). 

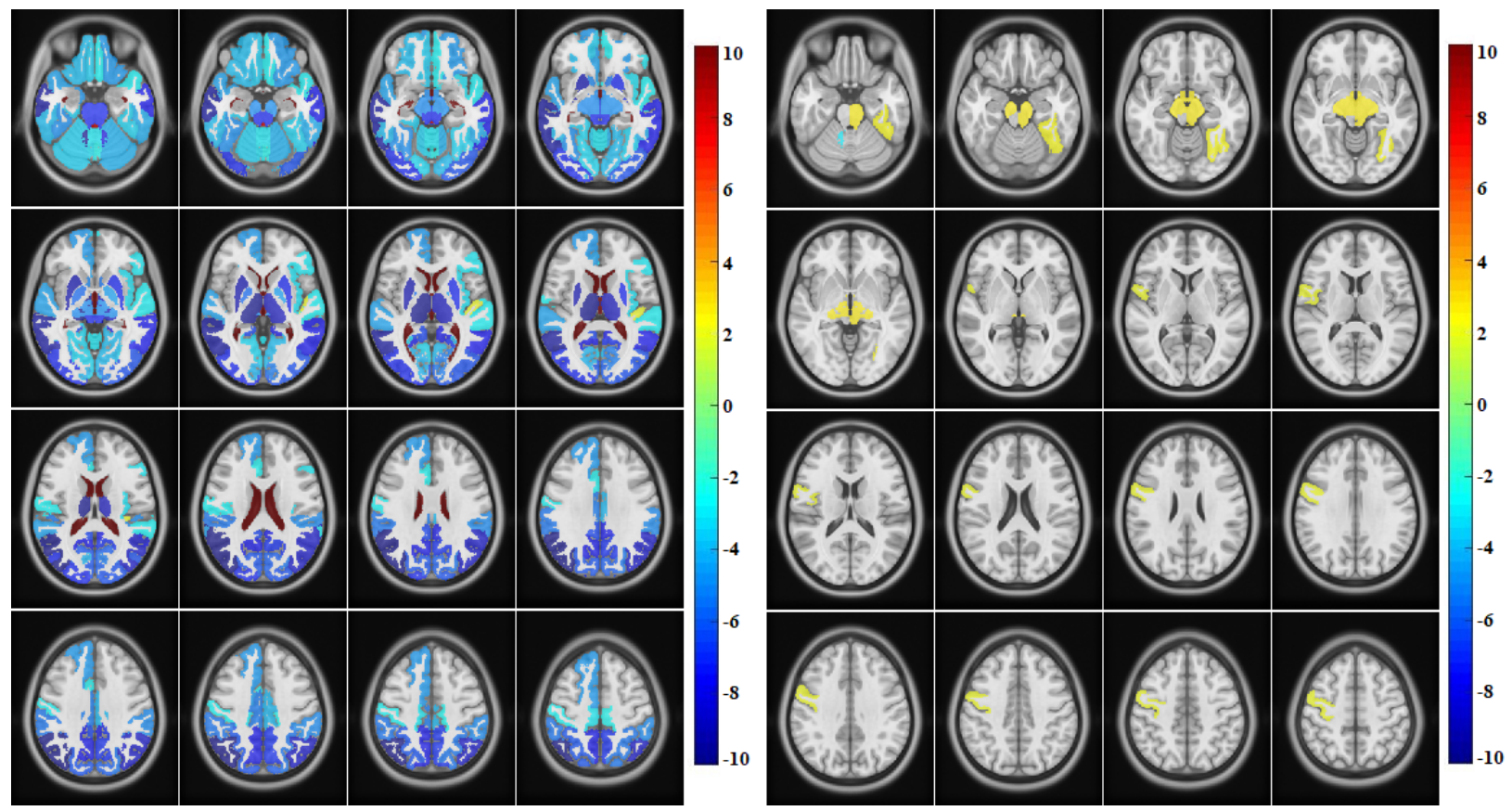

Figure 3: Left panel: t-statistical-map showing significant areas of atrophy associated to FWML volume increase. Right panel: t-statistical-map showing significant areas of atrophy associated to DAWM decrease.

\subsection{EDSS, SDMT, and 9HPT scores as a function of FWML and DAWM volumes}

Mixed effect models were used to assess the relationship between clinical variables and FWML and DAWM volumes, controlling for Age, Sex, and Treatment. Global EDSS scores were significantly and positively associated with FWML volumes $(p=0.002)$, but not with DAWM volumes. Cerebellar, brainstem, and visual functional systems scores (EDSS sub-scores) were significantly and positively associated with FWML volume $(\mathrm{p}=0.002, \mathrm{p}<0.0001, \mathrm{p}<0.0001$, respectively). Cerebral functional system score (EDSS sub-score) was significantly and positively associated with FWML volumes $(\mathrm{p}=0.002)$, and negatively with DAWM volumes $(\mathrm{p}=0.03)$. Other functional system scores (pyramidal, sensory, and bowel and bladder functional systems) showed no significant association with either FWML or DAWM volumes (Figure 4). 
The 9HPT time was significantly and positively associated with FWML, both in the dominant $(p<0.0001)$ and non-dominant hands $(p<0.0001)$. Significant and negative associations were also found for the dominant $(\mathrm{p}=0.02)$ and non-dominant $(\mathrm{p}=0.001)$ hand 9HPT results and the DAWM volumes. SDMT results showed a significant negative association only with FWML volumes $(\mathrm{p}<0.0001)$ (Figure 4).
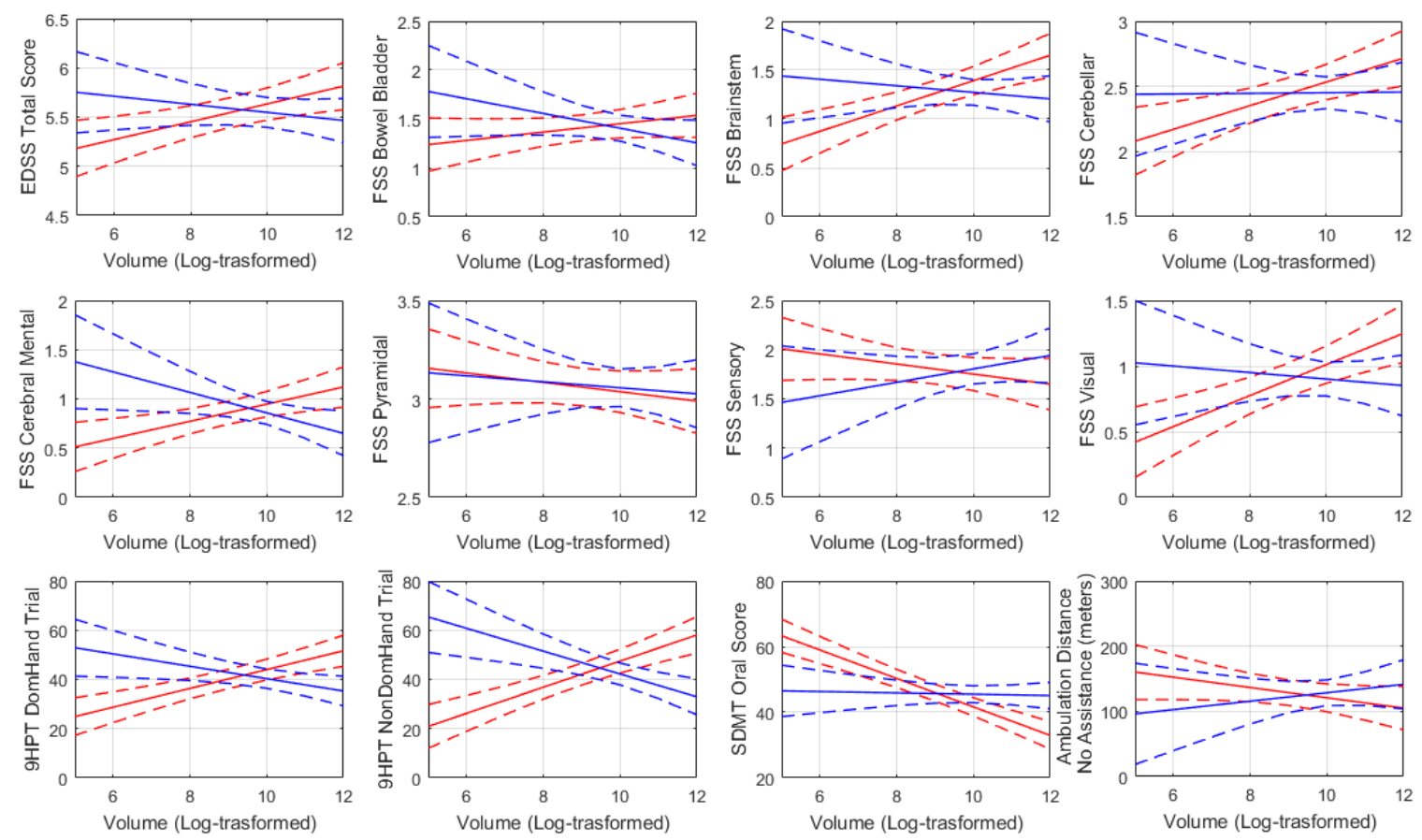

Figure 4: Association between DAWM and FWML volumes and clinical variables. Red lines: FWML volume. Blue lines: DAWM volumes.

\subsection{FWML and DAWM volumes and Progression}

CDP for 12 and 24 weeks showed a significant and positive association with increase of FWML volume during the course of the disease ( $<<0.0001$ for both 12 - and 24 -weeks). DAWM volumes were not significantly associated with progression. 


\subsection{Evolution of DAWM-to-FWML and Progression}

Voxels segmented as DAWM on the first scan of each participant that transformed to FWML on the last scan were considered as DAWM-to-FWML volumes and were significantly associated with progression $(12$-weeks $\mathrm{p}=0.01 ; 24$-weeks $\mathrm{p}<0.001)$. Additionally, the median volume of tissue that transformed from DAWM-to-FWML was significantly higher in patients with CDP for 24 weeks $(2.70 \mathrm{cc}$ vs $1.76 \mathrm{cc} ; \mathrm{p}<0.0001 ; \mathrm{F}=3.1)$, representing $14 \%$ of the total DAWM volume at screening, compared to $10 \%$ in patients who did not progress. Similar results were obtained patients with CDP for 12 weeks (2.61cc vs $1.75 \mathrm{cc} ; \mathrm{p}<0.0001 ; \mathrm{F}=1.5)$, representing $14 \%$ of the total DAWM volume at screening, compared to $10 \%$ in patients who did not progress.

Similarly, voxels of NAWM on the first scan of each participant that transformed to FWML on the last scan, NAWM-to-FWML volumes, were significantly higher in patients with CDP (12weeks $\mathrm{p}=0.004 ; \mathrm{F}=0.5 ; 24$-weeks $\mathrm{p}=0.003 ; \mathrm{F}=0.4$ ). Also, voxels of NAWM on the first scan of each participant that transformed to DAWM on the last scan, i.e. NAWM-to-DAWM volumes, were significantly higher in patients with $\mathrm{CDP}$ at 24 weeks $(\mathrm{p}=0.01 ; \mathrm{F}=0.06)$, but they did not reach significance for CDP at 12 weeks $(\mathrm{p}=0.06 ; \mathrm{F}=0.001)$.

Conversely, voxels of DAWM that transformed into NAWM, or FWML that transformed into NAWM or DAWM did not show a significant association with progression.

Interestingly, visual inspection of the longitudinal changes in the DAWM and FWML regions showed that the areas of DAWM-to-FWML were mostly distributed in the perimeter of FWML, which is the reported location of activity of chronic active lesions ${ }^{24}$ (Figure 5). 


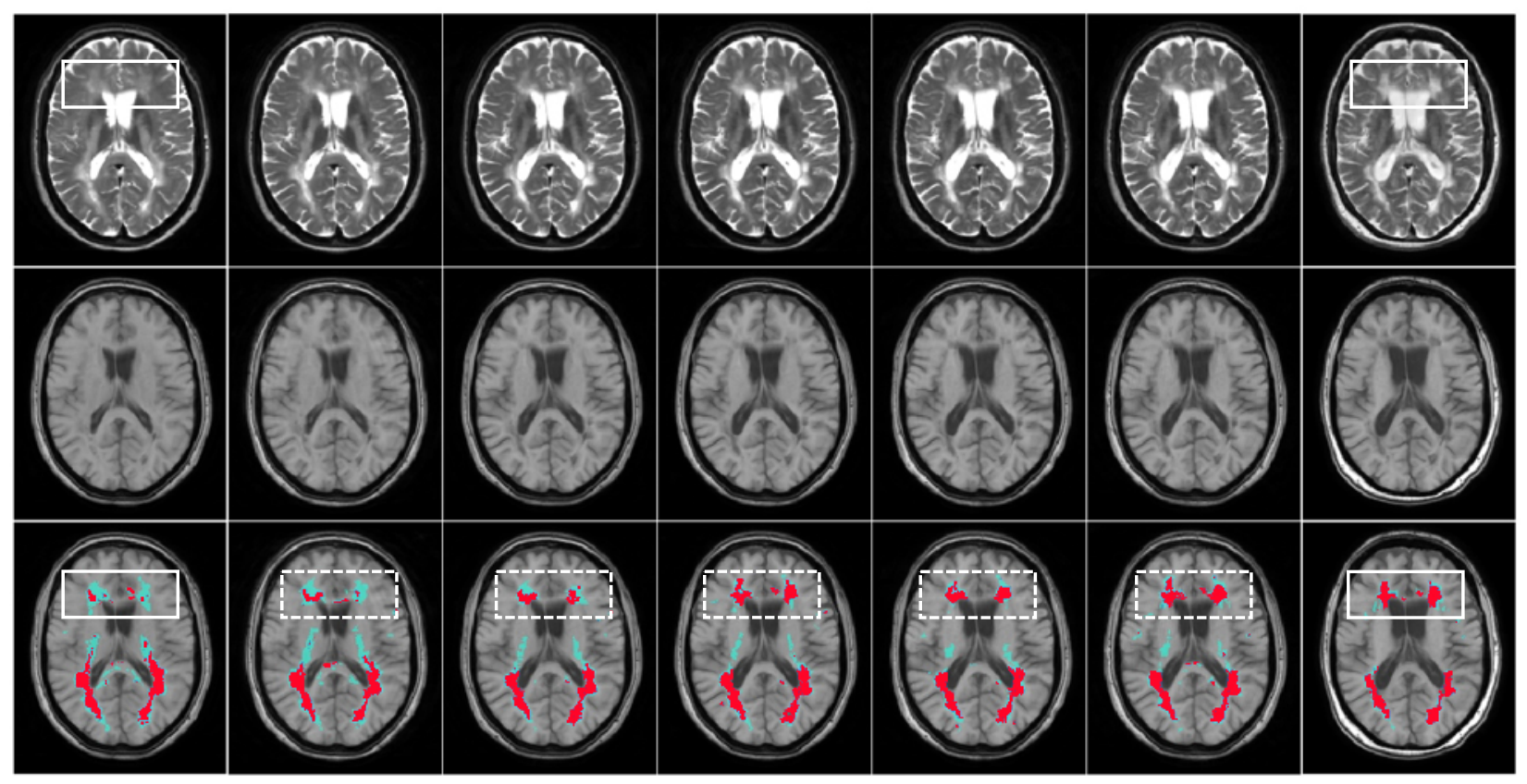

Figure 5: From left to right the columns represent the scans acquired at: screening, weeks 24, 48, 72, 108, 156. Top row: T2-weighted images. Mid row: T1-weighted images. Bottom row: T1weighted with masks overlaying areas of FWML (cyan) and DAWM (red). Frontal periventricular FWML areas expand at the expense of DAWM located around the focal lesion (white box).

Finally, when assessing the evolution of T1w-normalized intensities in DAWM-to-FWML areas, and NAWM-to-FWML, both showed a significant negative association with 12- and 24-weeks progression (DAWM-to-FWML 12-weeks progression: p=0.004; DAWM-to-FWML 24-weeks progression: $\mathrm{p}<0.00001$; NAWM-to-FWML 12-weeks progression: $\mathrm{p}<0.001$, and NAWM-toFWML 24-weeks progression: $\mathrm{p}<0.00001)$. T1w-noramalized intensities in NAWM-to-DAWM areas only showed a significant negative association with 24 -weeks progression $(\mathrm{p}=0.006)$. Association of NAWM-to-DAWM with 12-weeks progression did not reach significance $(\mathrm{p}=0.07)$. 


\section{DISCUSSION}

SPMS remains a form of MS that entails a poorer prognosis given the lack of response to most current available therapies that successfully control the focal inflammatory component of the disease $^{6,12}$. Different types of lesions, such as CLs ${ }^{25,26}$ and brain atrophy ${ }^{27,} 28,29$ have been proposed as being prominent in the progressive phase of MS, and hence considered as potentials predictors of fixed disability and progression. However, none have been identified as a definite predictive marker of progression. On one hand, CLs are difficult to identify on 3T MRI scans ${ }^{30,31}$, and require a highly experienced imaging expert to perform a very time-consuming manual segmentation. Additionally, 3T scans mainly capture a single type of CL, the leukocortical type, 32,33 and have very low sensitivity to detect subpial lesions ${ }^{32}$, the type that seems significantly associated with progression. In order to capture subpial lesions, $7 \mathrm{~T}$ scans are necessary ${ }^{32}$, but such ultra-high-field MRI scanners are found in only a few centers and usually only used for research purposes. For these reasons, CL quantification seems impractical, at least for now, as a marker of MS progression. Brain atrophy has been another useful measurement, extensively explored as a marker of fixed disability ${ }^{8}$, but its exclusive role in progression is still difficult to weigh, given that atrophy rates seem to be similar in all stages of MS, from the clinical isolated syndrome phase, to the SPMS stage ${ }^{34}$.

Histopathology studies have suggested that the diffuse WM lesion component, DAWM, could be related to progression ${ }^{7}$. Our study explored not only the characteristics of DAWM, but also its relation to FWML at various levels, and proposes some of these characteristics as potential useful markers of progression:

- FWML areas showed significant lower T1w-normalized intensity values than those of DAWM areas, suggesting that DAWM exhibits a lower level of demyelination ${ }^{12}$. 
- DAWM volume was significantly higher in patients that develop the disease at a younger age. In this respect DAWM did not differ from FWML (Figure 2). However, volume changes of FWML and DAWM were inversely related: as disease duration increased, DAWM decreased and FWML increased. We also calculated the ratio of FWML/DAWM volumes on a per-participant basis, in an attempt to assesses whether DAWM was turning into FWML on each individual case. We found a significant positive association of this ratio and disease duration.

- FWML volume increase and DAWM decrease were significantly associated to GM atrophy, with large areas of both, deep and cortical GM, related to FWML increase, and some areas of cortex and the brainstem related to DAWM decrease.

- FWML volume increase over time, but not DAWM decrease, was significantly associated to clinical variables such as EDSS and some of their sub-scores (Figure 4).

- FWML volume increase, but not DAWM decrease, was related to 12- and 24-week CDP.

Since the increase of FWML was more prominently associated with clinical variables, but, at the same time, the FWML/DAWM ratio suggested a change of DAWM into FWML volumes on a per-participant basis, we explored associations of voxels that were segmented as DAWM in the first scan and transformed to FWML in the last scan. We then related this volume (i.e. DAWMto-FWML volume) with progression. We found that DAWM-to-FWML volume was significantly higher in patients with CDP (for both 12- and 24-weeks). NAWM-to-FWML volume was also significantly higher in CDP (for both 12- and 24-weeks), although with lower F values than DAWM-to-FWML. NAWM-to-DAWM showed a significant difference only in participants with CDP for 24 weeks, and was marginally significance for CDP for 12 weeks $(p=0.06)$. These results suggest that conversion of DAWM to FWML seem to seal the progressive course. 
We also noted that the distribution of the DAWM-to-FWML voxels occurs in the perimeter of FWMLs, suggesting a potential overlap with areas that have been described as chronic active lesions by other groups ${ }^{24}$.

Finally, we explored the changes in T1w-normalized intensity values in the areas of DAWM-toFWML and NAWM-to-FWML. They both showed significant negative associations with 12- and 24-week CDP. Moreover, NAWM-to-DAWM T1w-normalized intensity values, also significantly decrease, showing a negative association with 24 -week progression. The progressive decrease of T1w-normalized values as the disease progresses and their significant association with progression highlights the impact of the severity of these lesions (e.g. stronger demyelination). To summarize, not only a significant quantitative volume change of DAWM-to-FWML and NAWM-to-FWML characterized patients who progress, but also a significant decrease in T1w-normlized values of these areas, which translates the qualitative aspect of the change: more severe demyelination in these areas.

Our study is not without limitations. There were no quantitative MRI sequences such as magnetization transfer ratio (MTR) images or diffusion available, to assess the nature of the changes in a given area in a more quantitative manner. However, previous studies have shown a significant association between MTR and T1w-normalized values ${ }^{12}$. Another limitation is the lack of scans of these participants during the relapsing-remitting course of the disease. We acknowledge the possibility of different types of changes for DAWM and/or FWML, earlier in the course of the disease.

\section{CONCLUSION}

FWML volume was significantly associated with GM brain atrophy, 9HPT, SDMT, specific EDSS functional systems (e.g. cerebellar), and progression in this group of SPMS participants. 
DAWM transformed into FWML over time, and this transformation was significantly associated with clinical progression. DAWM voxels that transformed had greater normalized T1w intensity decrease over time, in keeping with relatively greater tissue damage evolution. Therefore, evaluation of DAWM in progressive MS may provide a useful measure for therapies that aim to protect this at-risk tissue, which would have the potential to slow progression.

\section{REFERENCES}

1. Reich DS, Lucchinetti CF, Calabresi PA. Multiple Sclerosis. The New England journal of medicine 2018;378:169-180.

2. Kamm CP, Uitdehaag BM, Polman CH. Multiple sclerosis: current knowledge and future outlook. European neurology 2014;72:132-141.

3. Popescu BF, Pirko I, Lucchinetti CF. Pathology of multiple sclerosis: where do we stand? Continuum (Minneapolis, Minn) 2013;19:901-921.

4. Lublin FD. New multiple sclerosis phenotypic classification. European neurology 2014;72 Suppl 1:1-5.

5. Absinta M, Sati P, Reich DS. Advanced MRI and staging of multiple sclerosis lesions. Nature reviews Neurology 2016;12:358-368.

6. Tintore M, Vidal-Jordana A, Sastre-Garriga J. Treatment of multiple sclerosis - success from bench to bedside. Nature reviews Neurology 2019;15:53-58.

7. Kutzelnigg A, Lucchinetti CF, Stadelmann C, et al. Cortical demyelination and diffuse white matter injury in multiple sclerosis. Brain 2005;128:2705-2712.

8. Sastre-Garriga J, Pareto D, Rovira A. Brain Atrophy in Multiple Sclerosis: Clinical Relevance and Technical Aspects. Neuroimaging clinics of North America 2017;27:289-300. 
9. Laule C, Pavlova V, Leung E, et al. Diffusely abnormal white matter in multiple sclerosis: further histologic studies provide evidence for a primary lipid abnormality with neurodegeneration. Journal of neuropathology and experimental neurology 2013;72:42-52.

10. Laule C, Vavasour IM, Leung E, et al. Pathological basis of diffusely abnormal white matter: insights from magnetic resonance imaging and histology. Mult Scler 2011;17:144-150.

11. Seewann A, Vrenken H, van der Valk P, et al. Diffusely abnormal white matter in chronic multiple sclerosis: imaging and histopathologic analysis. Arch Neurol 2009;66:601-609.

12. Maranzano J, Dadar M, Arnold DL, Collins DL, Narayanan S. Automated Separation of Diffusely Abnormal White Matter from Focal White Matter Lesions on MRI in Multiple Sclerosis. BioRxiv 2019.

13. Kapoor R, Ho PR, Campbell N, et al. Effect of natalizumab on disease progression in secondary progressive multiple sclerosis (ASCEND): a phase 3, randomised, double-blind, placebo-controlled trial with an open-label extension. The Lancet Neurology 2018;17:405-415.

14. Sled JG, Zijdenbos AP, Evans AC. A nonparametric method for automatic correction of intensity nonuniformity in MRI data. IEEE transactions on medical imaging 1998;17:87-97.

15. Eskildsen SF, Coupe P, Fonov V, et al. BEaST: brain extraction based on nonlocal segmentation technique. Neuroimage 2012;59:2362-2373.

16. Dadar M. CD. BISON: Brain tISue segmentatiON pipeline using T1-weighted magnetic resonance images and a random forests classifier. BioRxiv 2019.

17. Dadar M, Fonov VS, Collins DL. A comparison of publicly available linear MRI stereotaxic registration techniques. Neuroimage 2018;174:191-200.

18. Ashburner J, Neelin P, Collins DL, Evans A, Friston K. Incorporating prior knowledge into image registration. Neuroimage 1997;6:344-352. 
19. Klein A, Tourville J. 101 labeled brain images and a consistent human cortical labeling protocol. Frontiers in neuroscience 2012;6:171.

20. Manera AL DM, Collins DL, Ducharme S. Deformation Based Morphometry Study of Longitudinal MRI Changes in Behavioral Variant Frontotemporal Dementia. BioRxiv 2019.

21. Elliott C. A Bayesian framework for 4-D segmentation of Multiple Sclerosis lesions in serial MRI in the brain: McGill University, 2016.

22. Zeighami $Y$, Ulla M, Iturria-Medina $Y$, et al. Network structure of brain atrophy in de novo Parkinson's disease. eLife 2015;4.

23. Genovese CR, Lazar NA, Nichols T. Thresholding of statistical maps in functional neuroimaging using the false discovery rate. Neuroimage 2002;15:870-878.

24. Elliott C, Wolinsky JS, Hauser SL, et al. Slowly expanding/evolving lesions as a magnetic resonance imaging marker of chronic active multiple sclerosis lesions. Mult Scler 2018:1352458518814117.

25. Kutzelnigg A, Lassmann H. Cortical demyelination in multiple sclerosis: a substrate for cognitive deficits? J Neurol Sci 2006;245:123-126.

26. Bo L, Vedeler CA, Nyland HI, Trapp BD, Mork SJ. Subpial demyelination in the cerebral cortex of multiple sclerosis patients. Journal of neuropathology and experimental neurology $2003 ; 62: 723-732$.

27. Popescu V, Agosta F, Hulst HE, et al. Brain atrophy and lesion load predict long term disability in multiple sclerosis. J Neurol Neurosurg Psychiatry 2013;84:1082-1091.

28. Dekker I, Eijlers AJC, Popescu V, et al. Predicting clinical progression in multiple sclerosis after 6 and 12 years. European journal of neurology 2019;26:893-902. 
29. De Stefano N, Airas L, Grigoriadis N, et al. Clinical relevance of brain volume measures in multiple sclerosis. CNS drugs 2014;28:147-156.

30. Geurts JJ, Roosendaal SD, Calabrese M, et al. Consensus recommendations for MS cortical lesion scoring using double inversion recovery MRI. Neurology 2011;76:418-424.

31. Maranzano J, Rudko DA, Arnold DL, Narayanan S. Manual Segmentation of MS Cortical Lesions Using MRI: A Comparison of 3 MRI Reading Protocols. AJNR Am J Neuroradiol 2016. 32. Maranzano J, Dadar M, Rudko DA, et al. Comparison of Multiple Sclerosis Cortical Lesion Types Detected by Multicontrast 3T and 7T MRI. AJNR Am J Neuroradiol 2019;40:1162-1169.

33. Nielsen AS, Kinkel RP, Tinelli E, Benner T, Cohen-Adad J, Mainero C. Focal cortical lesion detection in multiple sclerosis: 3 Tesla DIR versus 7 Tesla FLASH-T2. J Magn Reson Imaging 2012;35:537-542.

34. De Stefano N, Giorgio A, Battaglini M, et al. Assessing brain atrophy rates in a large population of untreated multiple sclerosis subtypes. Neurology 2010;74:1868-1876. 